\title{
Erratum
}

\section{Erratum to: Field dependence of magnetoresistance in half-metallic manganite}

\author{
Eur. Phys. J. Appl. Phys. (2015) 70: 30601
}

Zhengming Zhang ${ }^{1, \mathrm{a}}$, Xingchen Zhao ${ }^{2}$, and Weiping Zhou ${ }^{3}$

${ }^{1}$ Department of Physics, Nanjing Tech University, Nanjing, Jiangsu 211816, P.R. China

${ }^{2}$ Department of Physics and Astronomy, Texas A\&M University, College Station, TX 77843, USA

${ }^{3}$ National Laboratory of Solid State Microstructures, Nanjing University, Nanjing, Jiangsu 210093, P.R. China

Received: 26 November 2017 / Accepted: 29 November 2017

This article contains a typographical error in the third paragraph of the subsection " 2.2 Grain boundary resistivity" on page 30601-p3, where a minus sign was omitted. The correct version appears below

"The equilibrium configuration of the spins is determined by $\partial \gamma_{\text {total }} / \partial \phi_{1}=\partial \gamma_{\text {total }} / \partial \phi_{2}=0$, where $\phi_{1}=-\phi_{2}$ for symmetry reasons."

Cite this article as: Zhengming Zhang, Xingchen Zhao, Weiping Zhou, Erratum to: Field dependence of magnetoresistance in half-metallic manganite, Eur. Phys. J. Appl. Phys. 80, 30602 (2017) 